\title{
Assessment of reverberation time by two measurement systems for room electromagnetics analysis
}

\author{
Aliou Bamba*, Wout Joseph, David Plets \\ Emmeric Tanghe, Günter Vermeeren, Luc Martens \\ Ghent University/IBBT, \\ Department of Information Technology \\ Gaston Crommenlaan 8 bus 201 \\ B-9050 Ghent/Belgium \\ Email: aliou.bamba@intec.ugent.be
}

\author{
Jørgen B. Andersen, Jesper Ø. Nielsen \\ Department of Electronic Systems, Aalborg University \\ Niels Jernes Vej 12, 9220 Aalborg, Denmark \\ Email: jba@es.aau.dk
}

\begin{abstract}
A closed room environment is viewed as a lossy cavity, characterized by possibly a line of sight (LOS) component and diffuse scattering parts from walls and internal obstacles. A theory used in acoustics and reverberation chambers is applied for the electromagnetics case, and main issues related to measurement systems and antennas characteristics are discussed. The goal of this paper is the assessment of the reverberation time in an environment with different measurement systems. From the reverberation time one can derive the absorption area and hence the absorption cross section for humans in realistic environments.
\end{abstract}

Keywords: diffuse scattering, room electromagnetics, reverberation time, absorption cross section

\section{INTRODUCTION}

Indoor microwave propagation has been treated in detail for a number of years. More recently, "room electromagnetics" has become appealing because it does not require full knowledge of all details of the propagation environment [1], which makes it less tricky. A very simple propagation model with only few parameters is obtained by considering the indoor environment as a lossy cavity where all the effective losses can be described with a single parameter. The theory of wideband propagation in an environment is applied, similar to studies in acoustics and reverberation chambers. The acoustics community has been applying the method [2] since the 1920's (Sabine's equation), but the fundamental differences to the radio case is the polarization [3]. The basic model is very simple and considers a first arriving LOS (LineOf-Sight) signal if present and after that multiple reflections and scatterings giving rise to a tail with exponential decay and a time constant noted as the "reverberation time", similar to the acoustics case. Room electromagnetics theory is discussed in [3].

[1], [3], [4] investigated the reverberation time assessment using a channel sounder. In this paper the reverberation time assessment is compared for two different measurement systems, namely the channel sounder and the virtual channel sounder. The reverberation time assessment is of main importance because it is a function of the absorption area in the room [4] i.e., the total surface being absorbed radiation, and hence linked to the absorption cross section i.e., the body surface area being exposed to a radiation, for humans in realistic environments and other lossy objects.
First the two measurement systems configuration and environment are discussed in Section II. Section III describes the methodology to extract the "reverberation time" $(\tau)$, in Section IV we present the benchmarking results of both measurement systems and finally we conclude in Section $\mathrm{V}$

\section{CONFIGURATION AND ENVIRONMENT}

\section{A. Virtual MIMO channel sounder set up}

The virtual channel sounder setup for the MISO (Multiple Input Single Output) measurements is shown in Fig. 1.

A network analyzer (Rohde \& Schwarz ZVR) is used to measure the complex channel frequency response for a set of transmitting and receiving antenna positions. The channel is probed in a $500 \mathrm{MHz}$ measurement bandwidth for central frequency of $2.3 \mathrm{GHz}$. As transmitting ( $\mathrm{Tx}$ ) and receiving $(\mathrm{Rx})$ antenna, broadband omnidirectional biconical antennas of type Electro-Metrics EM-6116 are used. To be able to perform measurements for large $\mathrm{Tx}$-Rx separations, one port of the network analyzer is connected to the Tx through a $\mathrm{RF} /$ optical link with an optical fiber of length $500 \mathrm{~m}$. The RF signal sent into the $\mathrm{Tx}$ and the RF signal coming from the $\mathrm{Rx}$ are both amplified using an amplifier of type Nextec-RF NB00453 with an average gain of $37 \mathrm{~dB}$. The amplifiers assure

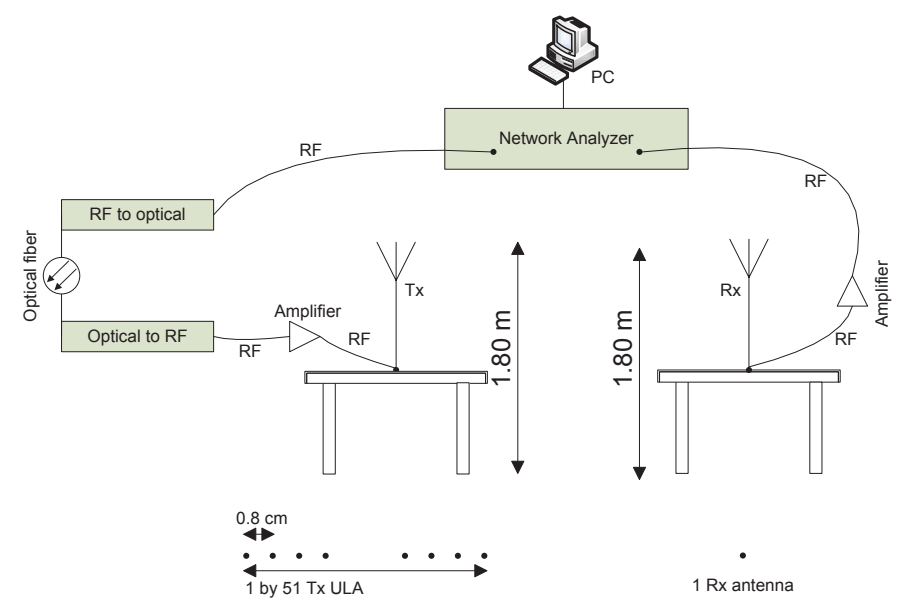

Figure 1. Virtual channel sounder setup

that the signal-to-noise ratio at the receiving port of the 
network analyzer is at least $20 \mathrm{~dB}$ for each measured location of the Tx and Rx. The calibration of the network analyzer is done at the connectors of the Tx and Rx antenna, and as such includes both the RF/optical link and the amplifiers.

Tx is moved along 1 by 51 virtual uniform linear arrays (ULAs), $\mathrm{Rx}$ is an 1 by 1 antenna. Both antennas are polarized vertically and positioned at a height of $1.8 \mathrm{~m}$ during measurements. At each of $51(1 \times 51 \times 1 \times 1)$ combinations of Tx and Rx positioning along the ULAs (Fig. 1), the network analyzer measures the $S_{21}$ scattering parameter ten times (i.e., 10 time observations), which we average to obtain a PDP per Tx-Rx combination. Since we have $51 \mathrm{Tx}$ and $1 \mathrm{Rx}$, we average again the PDPs over the number of Tx positions and obtain an average PDP in $d B$ labelled as APDP and given by:

$$
\left.A P D P(t)\right|_{d B}=10 \log \left(\left|S_{21}^{a v}(t)\right|^{2}\right)
$$

where $S_{21}^{a v}(t)$ is the average PDP in linear domain over the number of Tx positions.

\section{B. Channel sounder setup}

The measurements were also carried out using a MultipleInput Multiple-Output (MIMO) channel sounder, based on the correlation principle. The system allows truly simultaneous measurement of all up to $8 \mathrm{Rx}$ branches and $16 \mathrm{Tx}$ branches. Details of the measurement system may be found in [5]. Some main characteristics of the setup used in this set of measurements are:

- Time-triggered measurements at $60 \mathrm{~Hz}$. This ensures proper sampling of the channel which is changing due to both movements of the Rx movement and other external changes.

- Bandwidth: about $100 \mathrm{MHz}$

- Pseudo noise sequence length: 4095

- Carrier frequency: $2.3 \mathrm{GHz}$ and $5.8 \mathrm{GHz}$ dual-band. Only the $2.3 \mathrm{GHz}$ is used in the current work.

- Measurement duration: MS measurements: 600 samples $(10 \mathrm{~s})$

- Rx channels: 4 mockup laptop arrays are measured simultaneously, each with 4 antennas (measured via switch). The laptop arrays have 2 top mounted and 2 side-mounted dipole-like antennas.

- Tx channels: 4 dual-band branches, 4 element linear array with vertically polarized elements.

\section{Description of the environment}

The measurements were performed in a seminar room of Aalborg University (AAU), shown in Fig. 2, where only the right part is used. The room is equipped with tables, chairs, and ordinary meeting room facilities. The considered locations in this room are summarized in Table I.

The Tx had a fixed position whereas the Rx occupies positions Rx1, Rx7, Rx12 and Rx18 as shown in Fig. 2. The measurements with the channel sounder occured in May, 2010, the ones with the virtual channel sounder were performed from september $20^{t h}$ to september $24^{t h}, 2010$.

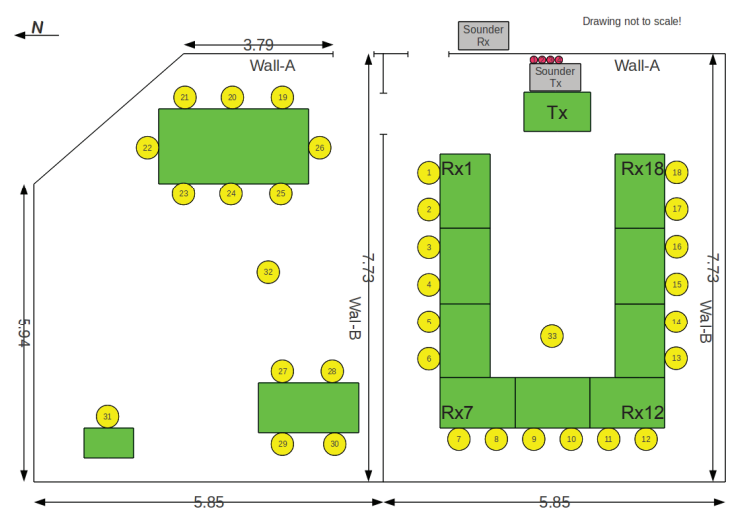

Figure 2. Seminar room: Tx and Rx measurement positions

\begin{tabular}{|c|c|c|}
\hline Position & Distance to Wall A (m) & Distance to Wall B (m) \\
\hline $\mathrm{R} x 1$ & 2.5 & 4.6 \\
\hline $\mathrm{R} x 7$ & 6.4 & 4.4 \\
\hline $\mathrm{R} 12$ & 6.5 & 1.4 \\
\hline $\mathrm{R} 1218$ & 2.5 & 1 \\
\hline
\end{tabular}

Table I

LOCATIONS USED IN THE SEMINAR ROOM

\section{Methodology}

\section{A. Extraction of $\tau$}

In indoor propagation, multiple reflections and scattering give rise to a tail with an exponential decay in the PDP curve and a time constant noted as the reverberation time [3]. In a log-scale this is equivalent to a tail of the impulse response which is linear, and its slope leads to the reverberation time according to the following formula:

$$
\tau=-\frac{10 \log (e)}{\text { slope }},
$$

where $e=2.718$ and slope is the slope of the linear tail in the impulse response.

The experimental APDP (in $\mathrm{dB}$ ) does not have actually a perfect linear tail. We need to derive the reverberation time over a certain delay range (or a certain range of power) for which the APDP tail is approximated by linear regression. This choice should be done automatically because the manual detection would suffer from judgement subjectivity. The methodology is shown in the flow graph of Fig. 3.

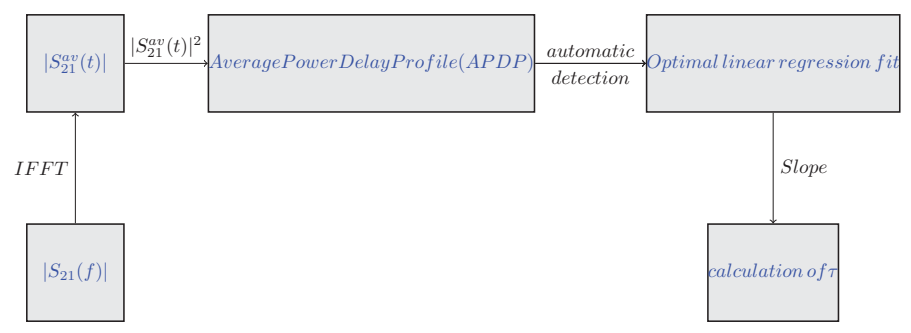

Figure 3. Flow chart of methodology for virtual channel sounder method

From the reverberation time [3] it is possible to assess the absorption area $\left(A^{\prime}\right)[4]$ (including walls, objects, possible 
people absorption) :

$$
A^{\prime}=\frac{4 V}{c \tau},
$$

where $V, c=3 \times 10^{8} \mathrm{~m} / \mathrm{s}$ and $\tau$ are the room volume, the light velocity and the reverberation time respectively.

If the absorption area is known with accuracy, it is possible to derive the absorption cross section and hence the whole body specific absorption rate (SAR) [6].

\section{B. Automatic detection algorithm}

In this section we introduce the algorithm (in Fig. 4) used for the automatic reverberation time detection. One may detect automatically the reverberation time using a fixed delay range (or a fixed power range). Such kind of algorithm would be good for a certain room and propagation environment. But when changing the room (mainly the volume), the Tx-Rx separation for the same room, or when the propagation environment changes (i.e., the visibility), the received power is different. Then the PDP tail varies and neither the previous delay range, nor the previous power range will be valid. The objective automatic algorithm is defined below.

We define here different quantities using the APDP curve (as in Fig. 4) to explain the algorithm:

- $T_{\text {mean }}$ : the mean arrival time,

- $P_{\min }$ : the APDP minimum value,

- $\Delta P_{n}$ : the difference between $P_{\min }$ and the first peak (local extremum) close to the minimum towards decreasing delays,

- $\Delta P_{p}$ : the difference between $P_{\min }$ and the first peak (local extremum) close to the minimum towards increasing delays,

The following threshold is then defined:

$$
\text { Threshold }=P_{\min }+\min \left(\Delta P_{n}, \Delta P_{p}\right),
$$

where $\min (a, b)$ is the minimum value between $a$ and $b$, we introduce $\Delta P_{n}$ and $\Delta P_{p}$ to ensure that the Threshold will be in the horizontal fluctuation region which we assume to be the noise zone (see Fig. 4).

All APDP values lower than Threshold are gathered in an array, which we called "NoiseArray". We finally define the noise floor by:

$$
P_{\text {noise }}=\operatorname{mean}(\text { NoiseArray })+3 d B,
$$

where $3 d B$ is added to be 2 times above the noise mean value.

Let denote by $\tau_{\text {noise }}$ the delay corresponding to $P_{\text {noise }}$. The reverberation time is derived by performing a linear regression fit between $T_{\text {mean }}$ and $\tau_{\text {noise }}$ as shown in Fig. 4 .

\section{BENCHMARKING}

\section{A. Channel sounder vs Virtual channel sounder}

In order to validate the virtual channel sounder, we compare its results with the channel sounder (measurement system of AAU). The measurements campaign has been performed in the same room in Aalborg University with both measurement systems. The measurements have been done at different dates and the $\mathrm{Tx}$ and $\mathrm{Rx}$ locations have

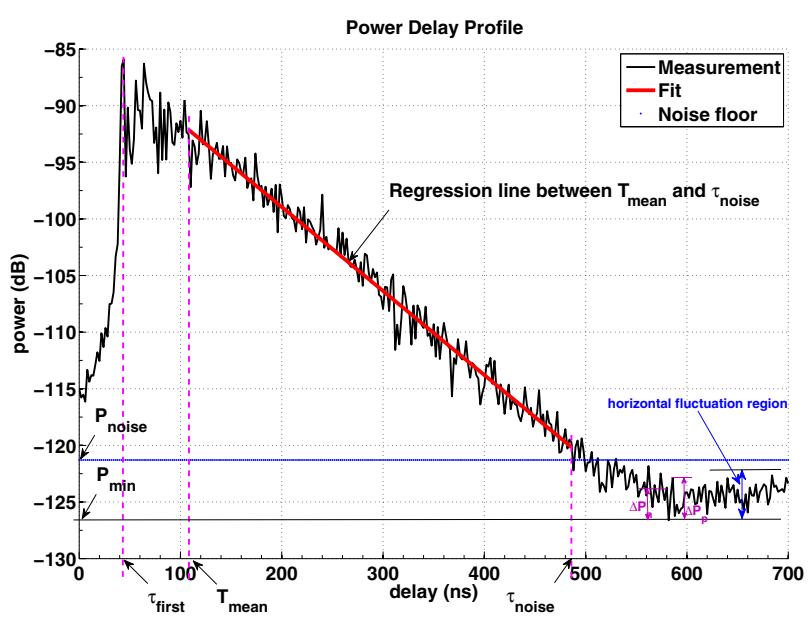

Figure 4. Power Delay Profile and explanation of automatic detection algorithm

been the same (as best as possible) for the two measurement campaigns.

Table II and III summarize the results for the reverberation time obtained for both measurement systems. $D, T_{\text {mean }}$, $T_{r m s}$ and $A^{\prime}$ are the Tx-Rx separation, the mean-delay arrival

\begin{tabular}{|c|c|c|c|c|c|c|}
\hline Position & $\begin{array}{l}\mathrm{D} \\
(\mathrm{m})\end{array}$ & Pers. & $\begin{array}{l}\tau \\
(\mathrm{ns})\end{array}$ & $\begin{array}{l}T_{\text {mean }} \\
\text { (ns) }\end{array}$ & $\begin{array}{l}T_{r m s} \\
\text { (ns) }\end{array}$ & $\begin{array}{l}A^{\prime} \\
\left(m^{2}\right)\end{array}$ \\
\hline $\mathrm{Rx} 1$ & 3.4 & 6 & 23.1 & 15.9 & 18.7 & 68 \\
\hline Rx7 & 4 & 6 & 22.7 & 18.2 & 18 & 69.3 \\
\hline $\mathrm{Rx} 12$ & 6.2 & 6 & 26.8 & 19.6 & 16.7 & 61.2 \\
\hline $\mathrm{Rx} 12$ & 6.2 & 9 & 25.5 & $\begin{array}{l}19.7 \\
\end{array}$ & 16.2 & 61.7 \\
\hline $\mathrm{Rx} 12$ & 6.2 & 12 & 25.3 & 20 & 15.7 & 62.1 \\
\hline Rx12 & 6.2 & 15 & 26.1 & 21 & 16.1 & 60.3 \\
\hline Rx18 & 2.7 & 12 & 25.3 & 20 & 15.7 & 62.1 \\
\hline
\end{tabular}
time of the rays, the root mean square arrival time, and the absorption area respectively.

PARAMETERS FROM CHANNEL SOUNDER MEASUREMENT

\begin{tabular}{|l|l|l|l|l|l|l|}
\hline Position & $\begin{array}{l}\mathrm{D} \\
(\mathrm{m})\end{array}$ & Pers & $\begin{array}{l}\tau \\
(\mathrm{ns})\end{array}$ & $\begin{array}{l}T_{\text {mean }} \\
(\mathrm{ns})\end{array}$ & $\begin{array}{l}T_{r m s} \\
(\mathrm{~ns})\end{array}$ & $\begin{array}{l}A^{\prime} \\
\left(\mathrm{m}^{2}\right)\end{array}$ \\
\hline $\mathrm{R} 1$ & 3.6 & 0 & 24.5 & 26.2 & 19 & 64.2 \\
\hline $\mathrm{R} 1$ & 3.6 & 2 & 23.8 & 26.2 & 18.7 & 66.2 \\
\hline $\mathrm{R} 77$ & 7.2 & 2 & 25.2 & 42.1 & 21 & 62.5 \\
\hline $\mathrm{R} 7$ & 7.2 & 4 & 25.1 & 40 & 19.9 & 62.7 \\
\hline $\mathrm{R} 7$ & 7.2 & 18 & 22 & 38.5 & 17.6 & 71.5 \\
\hline $\mathrm{R} 12$ & 6 & 2 & 28.2 & 39.5 & 22.3 & 55.8 \\
\hline $\mathrm{R} \times 18$ & 3 & 2 & 26 & 16.5 & 13.6 & 60.6 \\
\hline
\end{tabular}

Table III

PARAMETERS FROM VIRTUAL CHANNEL SOUNDER MEASUREMENT

As we are mainly interested in the reverberation time $\tau$, we focus the comparison on this parameter as illustrated in the diagram chart in Fig. 5. For the channel sounder we obtain a mean value of $\tau\left(\tau_{\text {mean }}\right)$ equal to $24.5 \mathrm{~ns}$ with a standard deviation $\sigma_{\tau}$ equal to $1.7 \mathrm{~ns}$ corresponding to $6.9 \%$ of $\tau_{\text {mean }}$. We obtain $\tau_{\text {mean }}=25.8 \mathrm{~ns}$ and $\sigma_{\tau}=1.6$ ns $\left(6.2 \%\right.$ of $\left.\tau_{\text {mean }}\right)$ for the virtual channel sounder. The deviation between reverberation time obtained with the two 


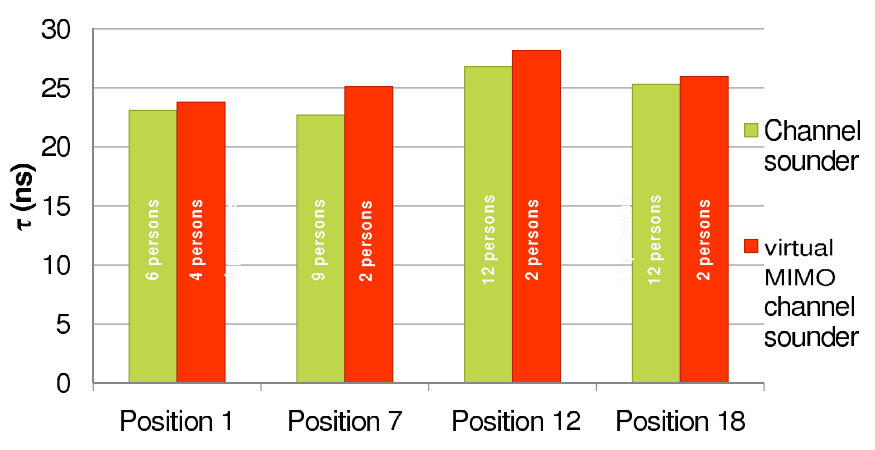

Figure 5. Channel sounder vs. virtual channel sounder measurement

measurement systems is only about $1.3 \mathrm{~ns}(5.2 \%$ of their mean value).

The observed few discrepancies between the two measurement systems may be due to the following reasons:

- differences between the measurement systems setup,

- variations in Tx-Rx positions, for a certain position the Tx-Rx separation may not be exactly the same for the two measurement campaigns (second column of Tables II and III).

- furnitures, tables, people, etc ... may not be in the same positions for both measurement campaigns,

- different number of people in the room as illustrated in the diagram chart (Fig. 5),

Given the small deviations between the reverberation time obtained for each measurement system, we can say that the theory holds - the reverberation time is independent of the transceiver locations in the room - whatever the measurement system used. However, we observe that the results obtained with the channel sounder are systematically lower than the ones obtained with the virtual channel sounder, this might be due to different polarization of the used antennas.

Good agreement between the two measurement systems is also obtained because of a deviation of only $1.3 \mathrm{~ns}$.

\section{B. Different antennas}

Using the virtual channel setup, we investigated the reverberation time variation using different antennas. We first use a broadband omnidirectional biconical antenna of type Electro-Metrics EM-6116 denoted "ibbt" and the "laptop antennas" mentionned in Section II-B denoted here as "aau". For the biconical antenna (ibbt) we obtain a mean value of

\begin{tabular}{|l|l|l|l|l|l|l|}
\hline Position & $\begin{array}{l}\mathrm{D}_{\text {ibbt }} \\
(\mathrm{m})\end{array}$ & $\begin{array}{l}\mathrm{D}_{\text {aau }} \\
(\mathrm{m})\end{array}$ & $\begin{array}{l}\text { Pers. } \\
(\mathrm{ibbt})\end{array}$ & $\begin{array}{l}\text { Pers. } \\
(\mathrm{aau})\end{array}$ & $\begin{array}{l}\tau_{(\text {ibbt })} \\
(\mathrm{ns})\end{array}$ & $\begin{array}{l}\tau_{(\text {aau })} \\
(\mathrm{ns})\end{array}$ \\
\hline $\mathrm{R} x 1$ & 3.6 & 3.6 & 1 & 1 & 24.1 & 24.9 \\
\hline $\mathrm{R} x 7$ & 7.2 & 6.6 & 2 & 1 & 25.2 & 23.7 \\
\hline $\mathrm{R} x 12$ & 6 & 6 & 2 & 1 & 28.2 & 25.8 \\
\hline $\mathrm{R} x 18$ & 3 & 2.4 & 2 & 1 & 26 & 22 \\
\hline
\end{tabular}

Table IV

PARAMETERS FOR DIFFERENT ANTENNAS USING THE VIRTUAL CHANNEL SOUNDER, IBBT $=$ BICONICAL ANTENNA AND AAU $=$ LAPTOP ANTENNA

$\tau\left(\tau_{\text {mean }}\right)$ about 25.9 ns whereas we obtain $\tau_{\text {mean }}=24.1$ ns for the laptop antenna, which gives a difference of $1.8 \mathrm{~ns}$ ( $7.2 \%$ of their mean value). Table IV summarize the results for different antennas. Few variations between the results is observed, but this was expected due the reasons mentioned in Section IV-A except that we used the same measurement system. Again we find the same trend using one measurement system but with different antennas. This indicates that different polarization properties might influence slightly the reverberation time.

\section{CONCLUSION}

Reverberation time dependency on two measurement systems and different antennas has been discussed. We obtain results confirming that the reverberation time is location and measurement system independent. However, the antenna polarization properties might have a small influence on the reverberation time. Reverberation time of about $25 \mathrm{~ns}$ is obtained in the seminar room. Most important, it is possible to make use of a virtual channel sounder to measure the reverberation time, from which we derive the absorption area and hence the specific absorption rate for humans in realistic environment.

Despite the very good agreement during the comparisons, the algorithm may be enhanced by distinguishing completely the coherent part from the diffuse part by starting the regression line at the beginning of the diffuse part, or using the well known RIMAX algorithm [7] to calculate the slope of the diffuse part in the Power Delay Profile.

\section{ACKNOWLEDGMENT}

The research leading to these results has received funding from the European Union's Seventh Framework Programme ([FP7/2007-2013]) under grant agreement $\mathrm{n}^{\circ} 244149$.

Wout Joseph is a Post-Doctoral Fellow of the FWO-V (Research Foundation - Flanders).

\section{REFERENCES}

[1] Jørgen Bach Andersen, Kin Lien Chee, Martin Jacob, Gert Frølund Pedersen, Thomas Kürner. Reverberations and Absorptions in an Aircraft Cabin with Passengers. submitted for publication, 2010.

[2] Heinrich Kuttruff. Room Acoustics. Spon Press, 2000.

[3] J.Bach Andersen, J.Ø. Nielsen, G.F. Pedersen, G. Bauch, and M. Herdin. Room Electromagnetics. IEEE Antennas and Propagation Magazine, Vol.49, No. 2, April 2007.

[4] J. Bach Andersen, J. Ø. Nielsen, G. Frølund Pedersen. Measured absorption by persons in a room environment (DRAFT). Technical report, Aalborg University, 2010.

[5] Nielsen J. Ø., Andersen J.B., Eggers P.C.F., Pedersen G.F., Olesen K., Suda H. . Measurements of indoor $16 \times 32$ wideband MIMO channels at $5.8 \mathrm{GHz}$. Spread Spectrum Techniques and Applications, 2004 IEEE Eighth International Symposium on, pages pp. 864- 868, 30 Aug.-2 Sept. 2004.

[6] Akimasa Hirata, Yoshio Nagaya, Fujiwara Osamu, Tomoaki Nagaoka, Soichi Watanabe. Correlation between Absorption Cross Section and Body Surface Area of Human for Far-Field Exposure at $\mathrm{GHz}$ Bands. Electromagnetic Compatibility, 200\%. EMC $200 \%$. IEEE International Symposium on, pages 1-4, 9-13 July 2007.

[7] Andreas Richter. Estimation of Radio Channel Parameters: Models and Algorithms. PhD thesis, Fakultt fr Elektrotechnik und Informationstechnik der Technischen Universitt Ilmenau, 2005. 